\title{
Revisiting the Marrow Metabolic Changes after Chemotherapy in Lymphoma: A Step towards Personalized Care
}

\author{
Bingfeng Tang, ${ }^{1}$ Malaykumar M. Patel, ${ }^{2}$ Regina H. Wong, ${ }^{2,3}$ Daniel Wood, ${ }^{2}$ \\ Christiana O. Wong, ${ }^{2,4}$ Dafang Wu, ${ }^{2}$ Pek Lan Khong, ${ }^{5}$ and Ching Yee Oliver Wong ${ }^{2,6}$ \\ ${ }^{1}$ Department of Radiology, The Ohio State University College of Medicine, Columbus, OH 43210, USA \\ ${ }^{2}$ Department of Radiology, Oakland University William Beaumont School of Medicine, Royal Oak, MI 48073, USA \\ ${ }^{3}$ LSA, The University of Michigan, Ann Arbor, MI 48109, USA \\ ${ }^{4}$ Saybrook College, Yale University, New Haven, CT 06511, USA \\ ${ }^{5}$ Department of Diagnostic Radiology, University of Hong Kong, Hong Kong, China \\ ${ }^{6}$ Positron Diagnostic Center and Medical Cyclotron, Department of Nuclear Medicine, William Beaumont Hospital, 3601 W, \\ Thirteen Mile Road, Royal Oak, MI 48073, USA
}

Correspondence should be addressed to Ching Yee Oliver Wong, owong@beaumont.edu

Received 7 June 2011; Accepted 24 June 2011

Academic Editor: Jun Hatazawa

Copyright () 2011 Bingfeng Tang et al. This is an open access article distributed under the Creative Commons Attribution License, which permits unrestricted use, distribution, and reproduction in any medium, provided the original work is properly cited.

\begin{abstract}
Purpose. The aims were to correlate individual marrow metabolic changes after chemotherapy with bone marrow biopsy (BMBx) for its potential value of personalized care in lymphoma. Methods. 26 patients (mean age, $58 \pm 15 \mathrm{y} ; 13$ female, 13 male) with follicular lymphoma or diffuse large B-cell lymphoma, referred to FDG-PET/CT imaging, who had BMBx from unilateral or bilateral iliac crest(s) before chemotherapy, were studied retrospectively. The maximal standardized uptake value (SUV) was measured from BMBx site over the same area on both initial staging and first available restaging FDG-PET/CT scan. Results. $35 \mathrm{BMBx}$ sites in 26 patients were evaluated. 12 of 35 sites were BMBx positive with interval decrease in SUV in 11 of 12 sites (92\%). The remaining 23 of 35 sites were BMBx negative with interval increase in SUV in 21 of 23 sites (91\%). The correlation between SUV change over the BMBx site before and after chemotherapy and BMBx result was significant $(P<0.0001)$. Conclusions. This preliminary result demonstrates a strong correlation between marrow metabolic changes (as determined by FDG PET) after chemotherapy and bone marrow involvement proven by biopsy. This may provide a retrospective means of personalized management of marrow involvement in deciding whether to deliver more extended therapy or closer followup of lymphoma patients.
\end{abstract}

\section{Introduction}

Molecular imaging using 2-deoxy-2-[F-18]fluoro-d-glucose (FDG) positron emission tomography (PET) scanning has recently emerged as a major imaging modality for the staging and followup of patients with non-Hodgkin's lymphoma (NHL) [1, 2]. Diffuse large B-cell lymphoma is the most common subtype. Follicular lymphoma accounts for $22 \%$ of NHL in adults with high tendency to involve the bone marrow [3, 4]. Follicular grade I lymphoma is the most predominating histological subtype to involve the marrow [5]. Before initiation of treatment, distinguishing potentially curable (stage I/II) from advanced disease (stage III/IV) may guide the planning of management. The advanced stages III and IV correlate significantly with shorter overall or event-free survival, and treatment may have to be modified accordingly. In NHL, bone marrow involvement places the patient in the advanced disease (stage IV). Bone marrow biopsy $(\mathrm{BMBx})$ is the established method for detection of bone marrow involvement in the initial staging and restaging of NHL. However, BMBx is a painful and invasive procedure and sometimes only a small sample can be obtained, which may be inconclusive due to sampling errors, despite bilateral iliac crest blind biopsy under anesthesia. Furthermore, even if the volume of the biopsy is adequate, focal lesions may be missed. Thus, although it is very specific, BMBx from 
traditional biopsy sites (iliac crests) has low sensitivity in detecting marrow involvement of lymphoma. It is essential to have a supplementary prospective, and if not possible even retrospective diagnostic procedure, possibly consisting of a multistep approach, to reliably assess bone marrow infiltration in patients with NHL to compliment the BMBx.

The ability of FDG-PET to evaluate bone marrow infiltration in patients with lymphoma has been investigated extensively. Multiple prior studies showed that FDG-PET has a high potential to detect bone marrow involvement in high-grade malignant lymphoma with low sensitivity for the detection of bone marrow infiltration in low-grade NHLs $[6,7]$ despite the fact that bone marrow involvement occurs in $30 \%-50 \%$ of patients with NHL. The majority of these studies used visual interpretation of marrow FDG uptake during whole-body staging PET scans to assess bone marrow involvement. Since it is more common in indolent histology $[8,9]$, the marrow activity may be not avid for visual detection based on a single staging PET scan. In the visual approach, the marrow was assumed to be abnormal where the FDG uptake was equal to or greater than uptake in the liver, which was usually greater than background. This approach for assessing marrow involvement depends on the extent of marrow infiltration by lymphoma and has made no use of available information from pre- and postchemotherapy intramedullary metabolic activities due to changes in cell population.

The standardized uptake value (SUV) is a semiquantitative measure of the glucose metabolism based on the degree of FDG uptake, which is derived from the tumor activity divided by dose per body mass in the attenuation-corrected PET images [10]. It may improve the definition of abnormal areas by reducing subjective assessment. It is common to see the pattern of diffusely increased FDG uptake in normal bone marrow after chemotherapy on F-18 FDG-PET scans due to change in hematopoietic cell population. Decreased FDG uptake after chemotherapy is noted in the areas with PET evidence of bone marrow involvement due to reduction in tumor population. The aims were to correlate individual marrow metabolic changes after chemotherapy with bone marrow biopsy (BMBx) for its potential value of personalized care in lymphoma.

\section{Materials and Methods}

Database of patients referred for FDG-PET/CT scan for initial staging and first restaging after chemotherapy who had BMBx from unilateral or bilateral iliac crest(s) before chemotherapy at a tertiary hospital and cancer center over a period of two years was retrospectively searched with Human Investigation Committee approval. Patients were excluded if they had malignancies other than lymphoma or if they had received prior radiation treatment or chemotherapy. Twentysix patients were eligible for this study. The mean age was 58 \pm 15 years old with 13 females and 13 males. There were 16 follicular lymphomas (FL, grades I, II, and III) and 10 diffuse large B-cell lymphomas (DLBC). The maximal standardized uptake value (SUV) was measured from BMBx site over the same area on both initial staging and restaging FDG-PET/CT scans. The interval changes of SUV were classified as decrease or increase and correlated with BMBx result of positive or negative for bone marrow involvement by lymphoma.

PET-CT imaging was obtained by a dedicated 16-slice body PET-CT scanner (GE Discovery DST, GE Medical Systems, Milwaukee, Wis, USA). All patients had fasted for 4 to 6 hours before the intravenous injections of average of $15 \mathrm{mCi}$ (555 MBq) F-18 FDG. PET scans were performed around one and a half hours after injection right after mapping CT. This choice of FDG uptake time was based on the modified schedule of usual one-hour time of FDG uptake to maximize the contrast between tumor, soft tissue, and benign inflammation if any [11]. The PET images were obtained at each bed position for 3 minutes with 6-8 beds to cover the entire body. The PET images were obtained using a two-dimensional high-sensitivity mode with an axial field of view of $15 \mathrm{~cm}$ in a $256 \times 256$ matrix. A 3-slice overlap was utilized between the bed positions. The PET images were reconstructed iteratively on a $128 \times 128$ matrix using ordered-subsets expectation maximization algorithm for 30 subsets and two iterations, with a $7.0 \mathrm{~mm}$ postreconstruction filter. An initial scout scan was obtained first to define the imaging field for the CT component of image acquisition, which used the following imaging parameters: $140 \mathrm{kVp}$, 120-200 mA, 0.8 seconds per CT rotation, pitch $1.75: 1$, and detector configuration of $16 \times 1.25 \mathrm{~mm}, 3 \mathrm{~mm}$ slice thickness with oral contrast only.

The serum glucose in $\mathrm{mg} / \mathrm{dL}$ was recorded just before PET, and the maximum SUV, defined as tumor activity divided by dose injected per body mass, was measured by searching the maximum value within a volume of interest over BMBx sites (posterior iliac crests) by a nuclear medicine physician. Bone marrow involvement was defined by histopathologically proven bone marrow lymphoma infiltration from marrow biopsy. Statistical analysis was done using SPSS (SPSS Inc, Chicago, IL) for comparing the changes of marrow before and after chemotherapy. A $P$ value of $<0.05$ was considered significant in all tests.

\section{Results}

Thirty-five BMBx sites from 26 patients were evaluated. Twelve of 35 sites were BMBx positive with interval decrease in SUV in eleven out of the 12 sites (92\%) (Figure 1). For the 11 sites with true positive PET findings (decreased SUV on BMBx positive sites), the magnitude of SUV decrease ranged from 0.7 to 15.8 ( -27 to $-89 \%$ ) for an average $8.3 \pm 5.9$ $(-64 \pm 24 \%)$.

The remaining twenty-three of 35 sites were $\mathrm{BMBx}$ negative with interval increase in SUV in 21 of 23 sites (91\%) (Figure 2). In the 21 sites with true negative PET results (increased SUV on BMBx negative sites), the range of SUV increase was from 0.1 to $9.2(+5$ to $+575 \%)$, average $1.7 \pm$ $2.0(+106 \pm 128 \%)$.

The correlation between SUV change over the BMBx site before and after chemotherapy and BMBx result was 


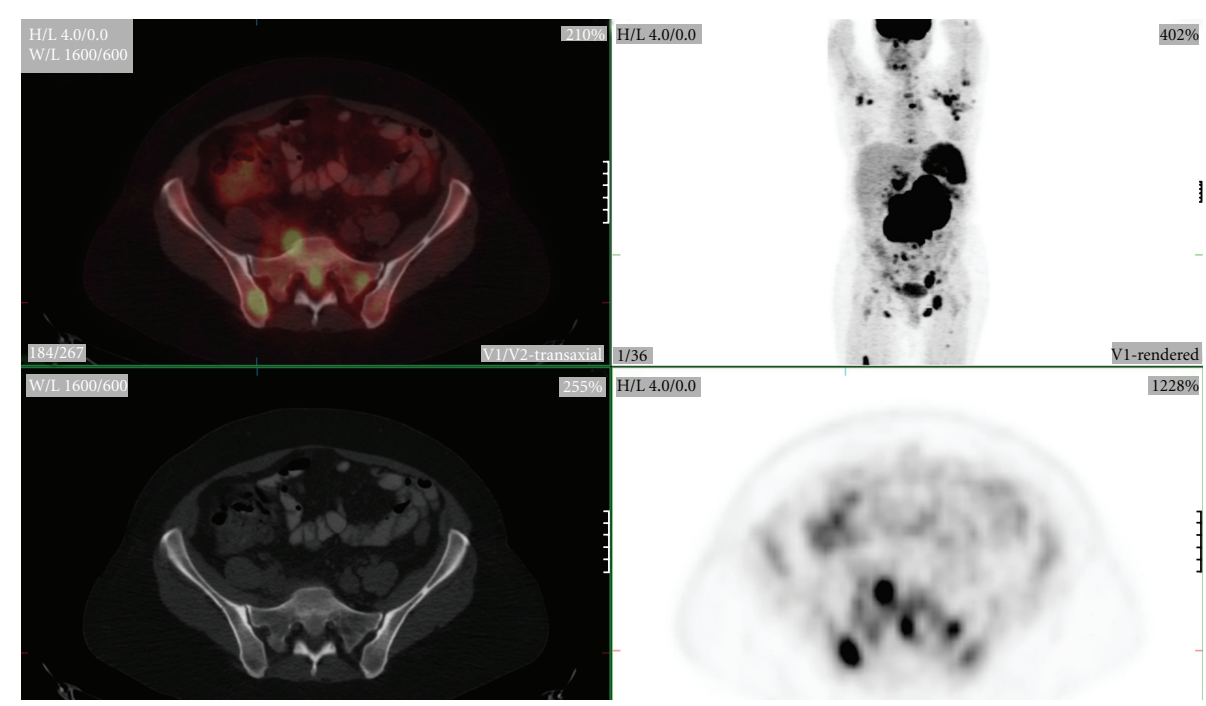

(a) Pretreatment PET-CT image

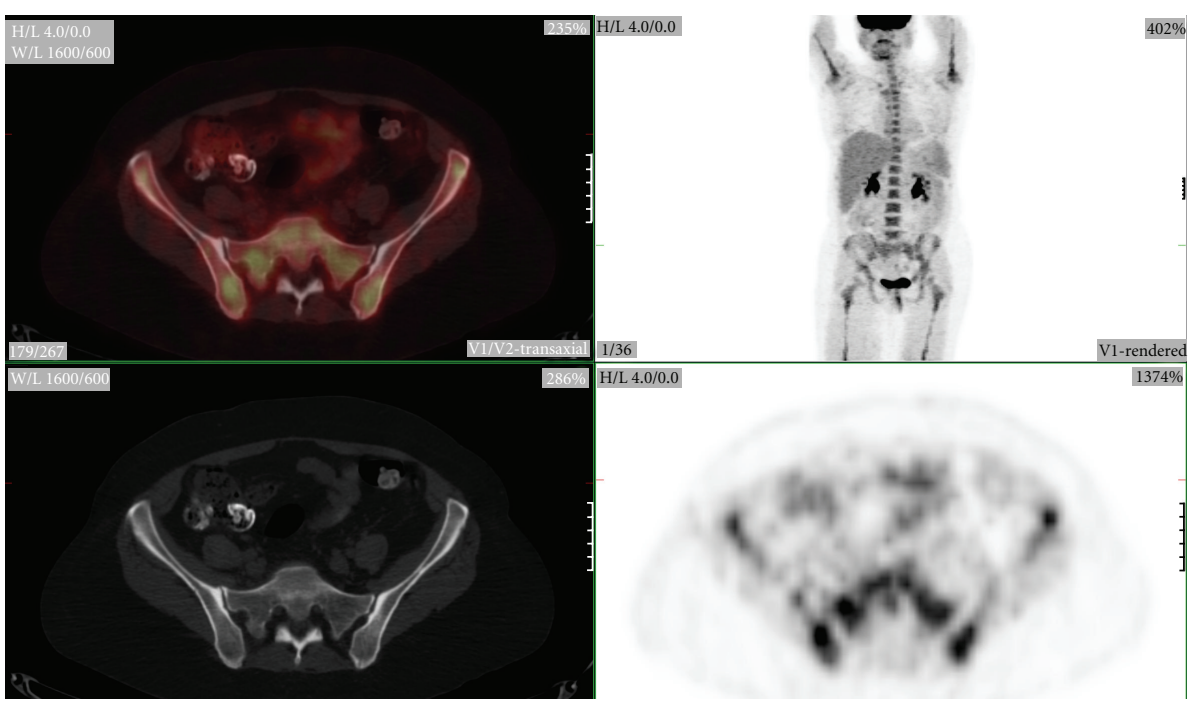

(b) Posttreatment PET-CT image

FIGURE 1: 53-year-old female with follicular grade 3 lymphoma with clear marrow involvement (a). This patient had bilateral iliac marrow biopsy. The post-treatment PET-CT (b) shows diffuse uniform increase in marrow uptake. However, the SUV actually decreases (from 6.7 to 4.9) on the right iliac bone marrow with pathologic confirmation of marrow involvement (b). On the contrary, the SUV over the pathologically negative left iliac bone marrow increases (from 2.7 to 4.2 ) due to normal hematopoietic response.

significant $(P<0.0001)$. The interval between chemotherapy and restaging PET scan was 9 to 75 days (mean $22 \pm 16$ days). The interval between BMBx and initial staging PET scan was 1 to 47 days (mean $8 \pm 9$ days).

\section{Discussion}

It is currently a common practice to use PET scan as a qualitative tool in the arena of lymphoma, supplemented by more clinically useful information extracted in the form of SUV, to aid in therapeutic decision making and prognostication. The clinical significance of the current study suggested that change in marrow SUV measurement after chemotherapy might provide a retrospective marrow staging, which may give the same information noninvasively as obtained from traditional $\mathrm{BMBx}$ regarding bone marrow involvement of lymphoma.

Bone marrow involvement in patients with lymphoma signifies extensive disease with less favorable prognosis. Although bone marrow biopsy is still the gold standard method for detection of bone marrow involvement in the initial staging or restaging of lymphomas, the potentially low sensitivity and invasive nature as well as other limitations of marrow biopsy make it nonideal diagnostic test in detection of marrow infiltration. A more reliable and sensitive noninvasive method of detecting marrow involvement would be desirable. FDG-PET imaging has become a major imaging modality for the staging and followup of patients with NHL 


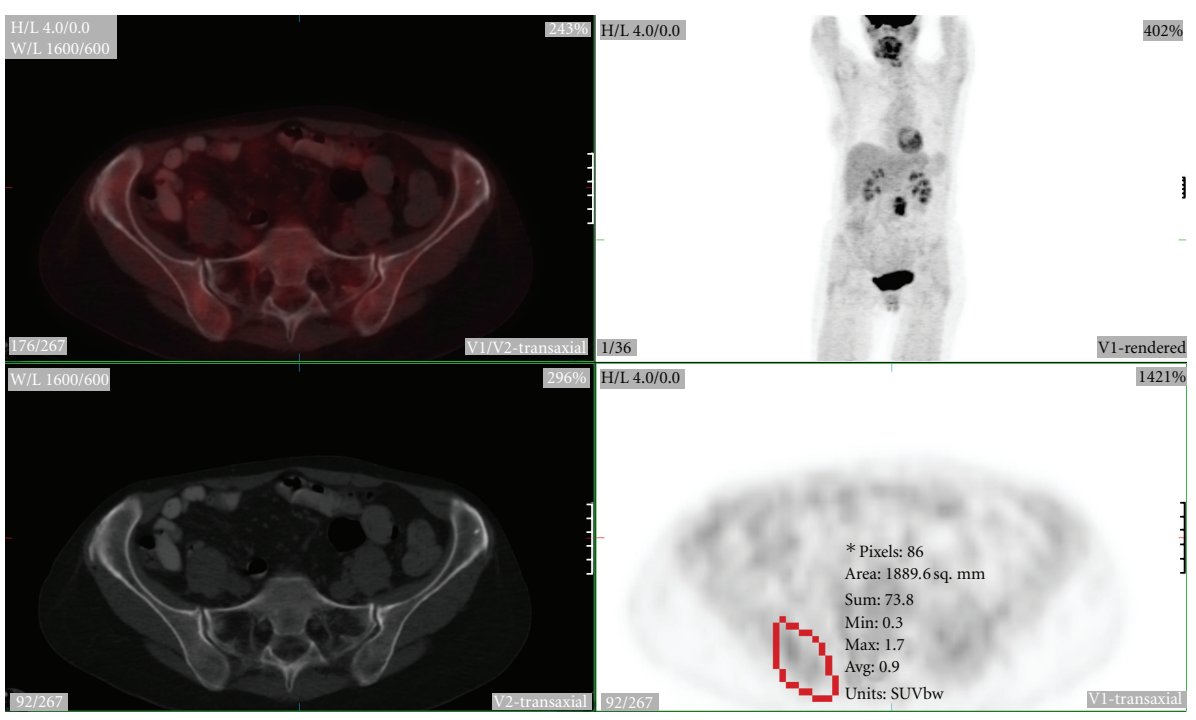

(a) Pretreatment PET-CT image

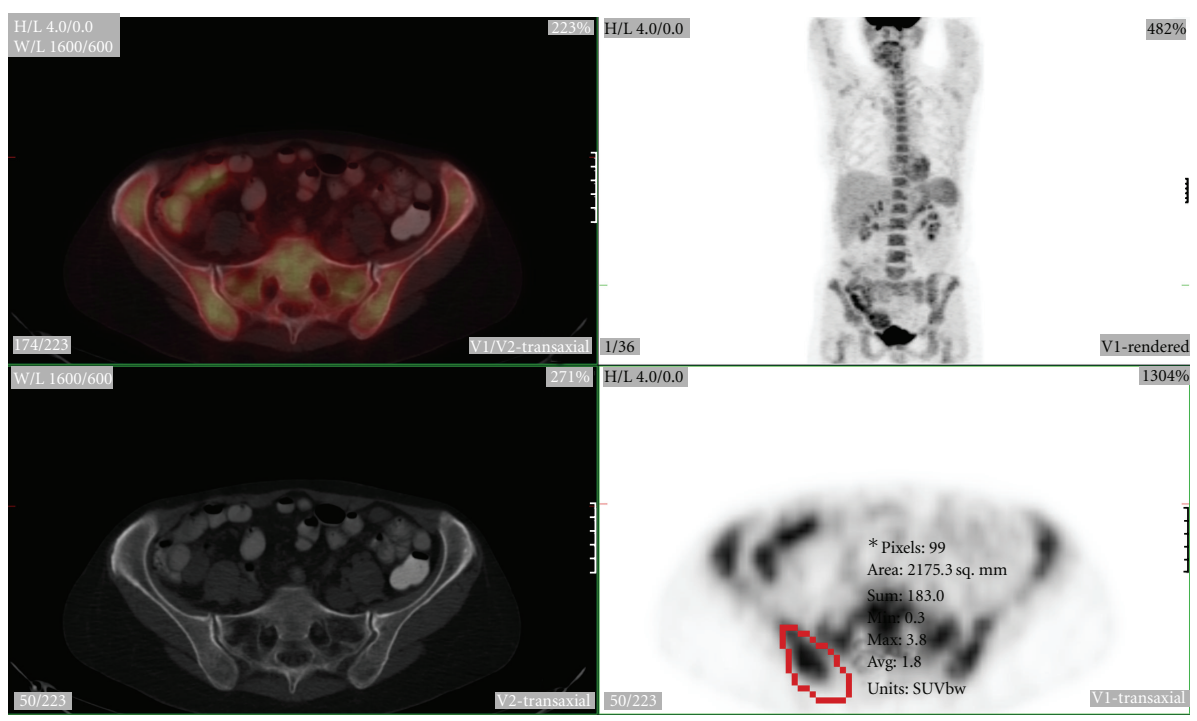

(b) Posttreatment PET-CT image

FIGURE 2: 58-year-old female with follicular grade 3 lymphoma (a) without focal evidence of marrow involvement by PET. She had right iliac biopsy. The posttreatment PET-CT (b) again shows diffuse increase in marrow uptake. The SUV increases (from 1.7 to 3.8 ) on the right iliac bone marrow (red region) with pathologic examination negative for marrow involvement. The change of the SUV over bone marrow is all due to normal hematopoietic response to chemotherapy.

$[1,2]$, and the potential ability of FDG-PET to evaluate both focal and diffuse bone marrow infiltration in patients with lymphoma is a natural choice for investigation and optimization. Though prior studies with pure visual interpretation of marrow FDG uptake to assess marrow have revealed a high potential to detect bone marrow involvement in malignant lymphoma [12-14], the sensitivity is still unacceptably low in low-grade non-Hodgkin's lymphomas $[6,7,15]$. The present study was undertaken to investigate and optimize the efficacy of FDG-PET as a potentially improved and complimentary method to aid in evaluating marrow involvement in patients for personalized care of lymphomas by utilizing individual interval metabolic changes instead of detection of focal abnormal marrow uptake based on a single staging PET scan alone.

The strong correlation between SUV change after chemotherapy and BMBx result as demonstrated from this current study has notable potential clinical significance. PET-CT is more sensitive than marrow biopsy and can be employed routinely to assess the entire marrow [16]. In patients with initial obvious focal FDG-avid bone marrow lesions, FDG-PET may offer guidance to biopsy or can be used as direct evidence of bone marrow involvement. The bone marrow involvement of these lesions can be confirmed by analyzing SUV changes after chemotherapy. If initial FDG PET shows no definite focal marrow lesions and patient 
needs chemotherapy clinically, then the retrospective analysis of SUV changes after chemotherapy over the traditional BMBx sites may offer similar if not better information as obtained from BMBx regarding bone marrow involvement by lymphoma and therefore potentially eliminate the error on management caused by false negative pretreatment $\mathrm{BMBx}$ due to sampling issue. Whether a blind marrow biopsy may still be warranted in patients with indolent lymphoma for whom chemotherapy may not be offered if there is no evidence of bone marrow involvement by lymphoma, or in patients with marrow population changes related to factors other than chemotherapy only, for example, GCSF treatment, bone marrow dysplasia, prior chemo- or radiation treatment, and so forth, needs further clinical investigation. Nonetheless, the current study moves a step towards personalized care by employing retrospective evidence of marrow involvement in lymphoma patients. Since the current revised response criteria for lymphoma require clearance of infiltrative marrow lesions by repeated biopsy [17], the current study may offer an alternative insight for noninvasive marrow response criteria by requiring involved marrow sites to show decrease in SUV from hot or normal FDG uptake to reduced or cold metabolic activity on the marrow.

With PET-CT, the functional PET images are coregistered with the anatomic CT images obtained by the almost simultaneously acquired CT scan. This approach can result in a significant improvement in the accurate anatomic localization and the region of interest determination and therefore make sure that the measurement of SUV is over the same area before and after chemotherapy. The current study demonstrates that the change in metabolic behavior correlates well with marrow cell population change and suggests possible optimization of metabolic information by using intramedullary SUV change to countercheck or supplement the invasive limited sampling of histological examination to predict marrow involvement, which in turn may lead to the most appropriate subsequent management for each individual patient.

There are limitations of this current retrospective study, including small sample size, mixed histopathological classification, and significant variation of interval between chemotherapy and restaging PET scan. A comparison between bone marrow biopsy and evaluations of FDGPET after treatment is not accomplished. This is partially because there will be little justification to repeat biopsy if there is already a good response to therapy. A welldesigned prospective study overcoming the above limitations to further confirm the conclusion of this preliminary study is necessary and is currently being investigated.

\section{Conclusion}

This preliminary study demonstrates a strong correlation between marrow metabolic changes by FDG PET scan after chemotherapy and marrow cell population change. There is an inverse relationship between (positive) marrow involvement of lymphoma and SUV change (decrease) after chemotherapy. The potential clinical significance of this observation is to provide a noninvasive retrospective means of countercheck or replacement of the invasive limited sampling of histological examination in predicting marrow involvement, which in turn may help in personalized care of deciding whether to deliver more extended therapy or closer followup of lymphoma patients.

\section{References}

[1] R. Kumar, I. Maillard, S. J. Schuster, and A. Alavi, "Utility of fluorodeoxyglucose-PET imaging in the management of patients with Hodgkin's and non-Hodgkin's lymphomas," Radiologic Clinics of North America, vol. 42, no. 6, pp. 10831100, 2004.

[2] C. Burton, P. Ell, and D. Linch, "The role of PET imaging in lymphoma," British Journal of Haematology, vol. 126, no. 6, pp. 772-784, 2004.

[3] The Non-Hodgkin's Lymphoma Classification Project, "A clinical evaluation of the international lymphoma study group classification of non-Hodgkin's lymphoma," Blood, vol. 89, no. 11, pp. 3909-3918, 1997.

[4] P. Lu, "Staging and classification of lymphoma," Seminars in Nuclear Medicine, vol. 35, no. 3, pp. 160-164, 2005.

[5] D. A. Arber and T. I. George, "Bone marrow biopsy involvement by non-Hodgkin's lymphoma: frequency of lymphoma types, patterns, blood involvement, and discordance with other sites in 450 specimens," American Journal of Surgical Pathology, vol. 29, no. 12, pp. 1549-1557, 2005.

[6] S. S. Foo, P. L. Mitchell, S. U. Berlangieri, C. L. Smith, and A. M. Scott, "Positron emission tomography scanning in the assessment of patients with lymphoma," Internal Medicine Journal, vol. 34, no. 7, pp. 388-397, 2004.

[7] E. E. Pakos, A. D. Fotopoulos, and J. P. A. Ioannidis, “18F-FDG PET for evaluation of bone marrow infiltration in staging of lymphoma: a meta-analysis," Journal of Nuclear Medicine, vol. 46, no. 6, pp. 958-963, 2005.

[8] K. Foucar, R. W. McKenna, G. Frizzera, and R. D. Brunning, "Bone marrow and blood involvement by lymphoma in relationship to the Lukes-Collins classification," Cancer, vol. 49, no. 5, pp. 888-897, 1982.

[9] M. G. Conlan, M. Bast, J. O. Armitage et al., "Bone marrow involvement by non-Hodgkin's lymphoma: the clinical significance of morphologic discordance between the lymph node and bone marrow. Nebraska Lymphoma Study Group," Journal of Clinical Oncology, vol. 8, no. 7, pp. 1163-1172, 1990.

[10] C. Y. O. Wong, J. Thie, K. J. Parling-Lynch et al., "Investigating the existence of quantum metabolic values in non-Hodgkin's lymphoma by F-18 FDG," Molecular Imaging and Biology, vol. 9, no. 1, pp. 43-49, 2007.

[11] C. Y. O. Wong, D. Noujaim, H. F. Fu et al., "Time sensitivity: a parameter reflecting tumor metabolic kinetics by variable dual-time f-18 FDG PET imaging," Molecular Imaging and Biology, vol. 11, no. 4, pp. 283-290, 2009.

[12] F. Moog, M. Bangerter, J. Kotzerke, A. Guhlmann, N. Frickhofen, and S. N. Reske, "18-F-fluorodeoxyglucose-positron emission tomography as a new approach to detect lymphomatous bone marrow," Journal of Clinical Oncology, vol. 16, no. 2, pp. 603-609, 1998.

[13] G. Jerusalem, Y. Beguin, F. Najjar et al., "Positron emission tomography (PET) with 18F-fluorodeoxyglucose (18FFDG) for the staging of low-grade non-Hodgkin's lymphoma (NHL)," Annals of Oncology, vol. 12, no. 6, pp. 825-830, 2001. 
[14] D. Fustera, S. Chianga, C. Andreadisb et al., "Can [18F]Fluorodeoxyglucose positron emission tomography imaging complement biopsy results from the iliac crest for the detection of bone marrow involvement in patients with malignant lymphoma?" Nuclear Medicine Communications, vol. 27, pp. 11-15, 2006.

[15] R. Elstrom, L. Guan, G. Baker et al., "Utility of FDG-PET scanning in lymphoma by WHO classification," Blood, vol. 101, no. 10, pp. 3875-3876, 2003.

[16] N. G. Schaefer, K. Strobel, C. Taverna, and T. F. Hany, "Bone involvement in patients with lymphoma: the role of FDGPET/CT," European Journal of Nuclear Medicine and Molecular Imaging, vol. 34, no. 1, pp. 60-67, 2007.

[17] B. D. Cheson, B. Pfistner, M. E. Juweid et al., "Revised response criteria for malignant lymphoma," Journal of Clinical Oncology, vol. 25, no. 5, pp. 579-586, 2007. 


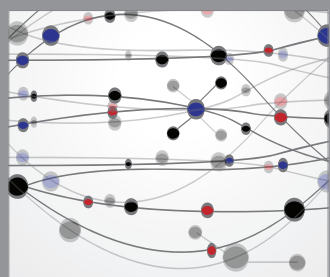

The Scientific World Journal
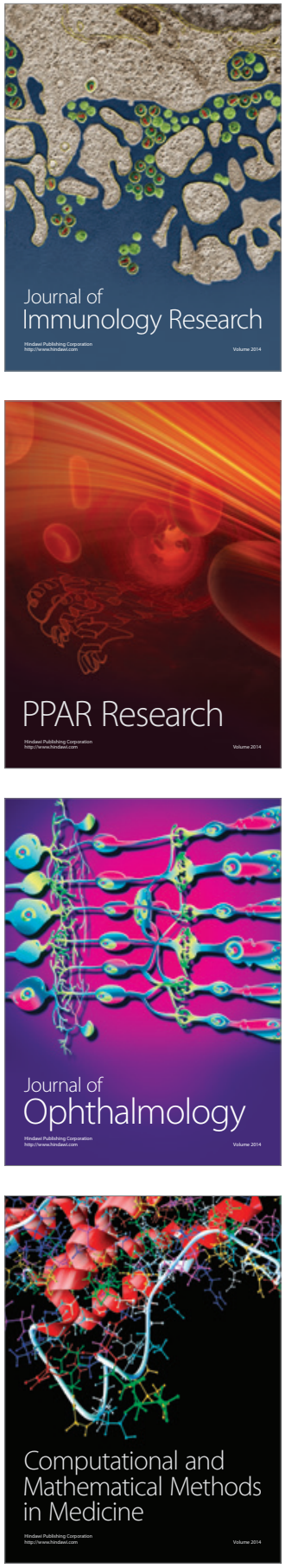

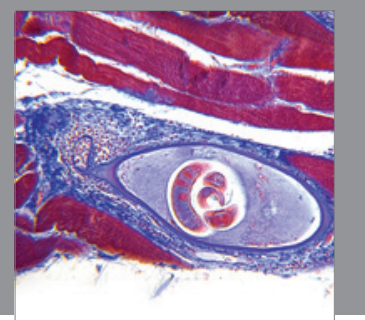

Gastroenterology

Research and Practice
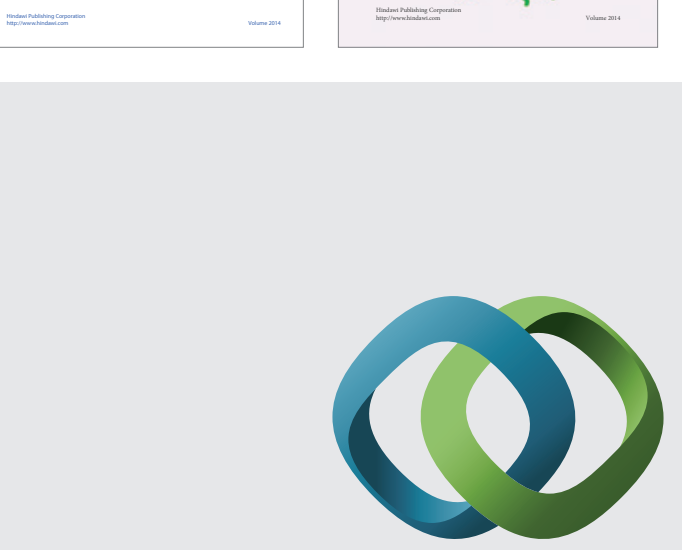

\section{Hindawi}

Submit your manuscripts at

http://www.hindawi.com
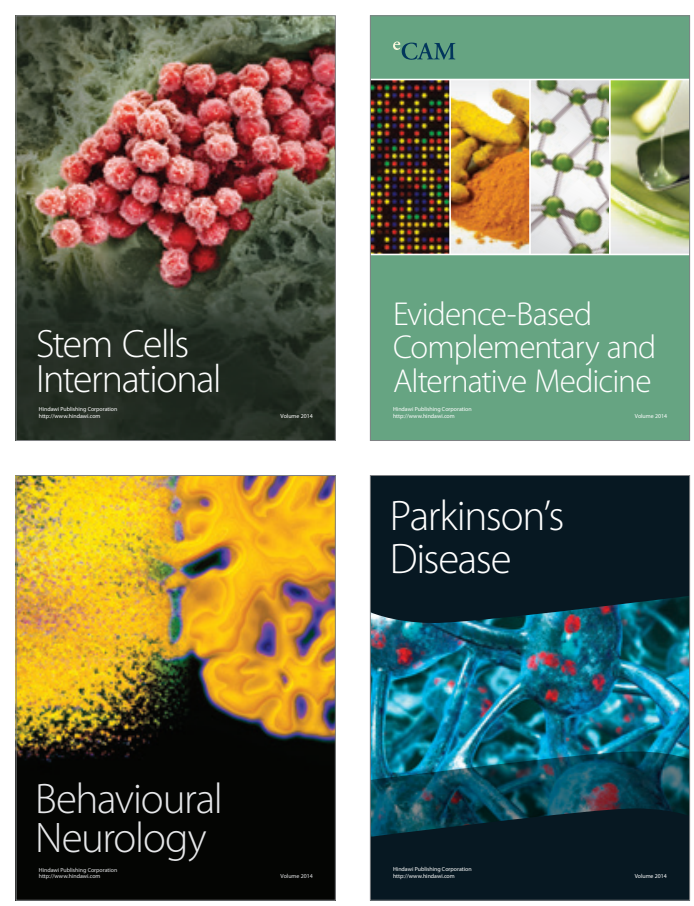

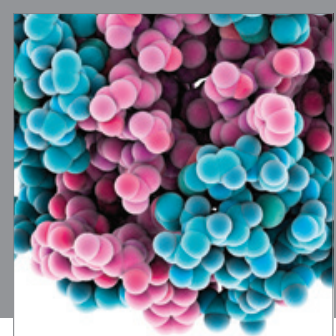

Journal of
Diabetes Research

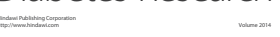

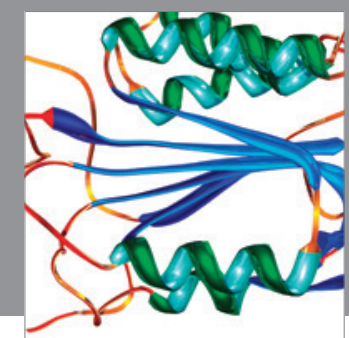

Disease Markers
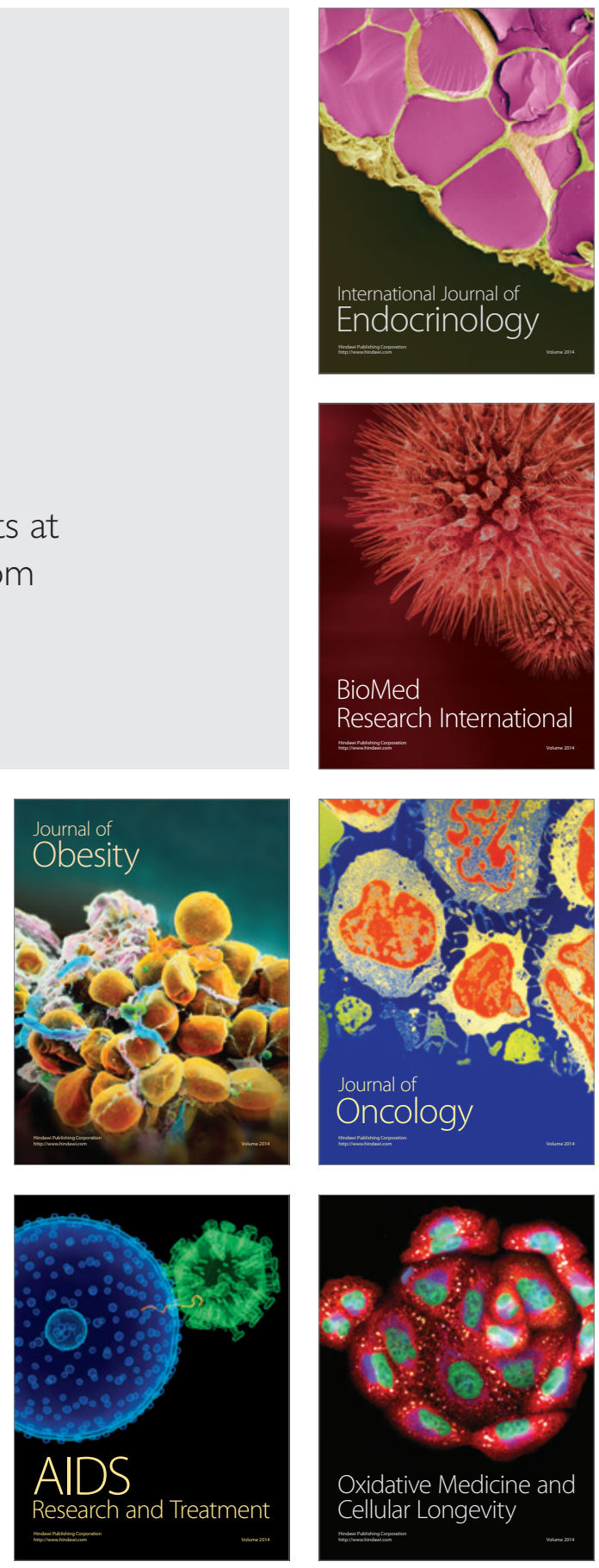